\section{Performance of 'Hamlin' Orange on 16 Rootstocks in East-central Florida}

\author{
Heinz K. Wutscher \\ U.S. Department of Agriculture, Agricultural Research Service, U.S. \\ Horticultural Research Laboratory, 2120 Camden Road, Orlando, FL 32803 \\ Laura Lee Hill \\ Citrus Grower, 5005 Lillian Lee Road, St. Cloud, FL 34771
}

Additional index words. tree growth, fruit production, fruit quality

\begin{abstract}
Hamlin' orange (Citrus sinensis L. Osbeck) was grown on 15 rootstocks: four citrumelos [C. paradisi Macf. x Poncirus trifoliata (L.) Raf.], five mandarin $\mathrm{x}$ trifoliate orange hybrids $(C$. reticulata Blanco $x P$. trifoliata), two pummelo $x$ trifoliate orange hybrids $[C$. grandis (L.) X P. trifoliata], Vangasay lemon ( $C$. limon Burm. f.), Norton citrange $(C$. sinensis $\times P$. trifoliata), and two Smooth Flat Seville $(C$. aurantium L. hybrid?) hybrids. These scion-rootstock combinations were compared to trees on Swingle citrumelo, the most widely used citrus rootstock in Florida. One Smooth Flat Seville hybrid was eliminated early because of poor growth and variability in size, and the Vangasay lemon rootstock was eliminated because of severe freeze damage. At age 5 , the trees on Norton citrange developed citrus blight and were eliminated. Remaining in the experiment for 7 years, 'Hamlin' trees on six of the 13 rootstocks produced more fruit than trees on Swingle citrumelo. Of these six, HRS 852 (Changsha mandarin x English large-flowered trifoliate orange) was the best overall rootstock, with trees on it producing large quantities of highquality fruit on medium-sized canopies.
\end{abstract}

Until the 1960s, the citrus rootstock situation in Florida had been stable for decades, with rough lemon used on deep sandy soils; sour orange on poorly drained flatwoods soils; and Cleopatra mandarin for tangelos, tangors, and other mandarin hybrids. Citrus blight outbreaks, a decline of yet undetermined cause, and more virulent tristeza virus disease in the 1980 s created a need for new rootstocks resistant to these diseases (Castle et al., 1993; Wutscher, 1988). Currently, the most planted rootstock is Swingle citrumelo, but it does not perform well on high-pH and clay soils (Jack Hebb, personal communication; Rouse and Wutscher, 1985). However, clay soils are common in limited areas in Florida. With the availability of many new hybrids from the U.S. Dept. of Agriculture breeding program (especially bred as rootstocks), new and better rootstocks are emerging from tests planted in the last 15 years. Our report compares the performance of 'Hamlin', the dominant early orange cultivar in Florida, on Swingle citrumelo with that of 'Hamlin' on 12 new rootstocks, one citrange, and two citrumelos little used in the past for this cultivar.

\footnotetext{
Received for publication 18 Apr. 1994. Accepted for publication 3 Oct. 1994. Mention of a trademark, warranty, proprietary product, or vendor does not constitute a guarantee by the U.S. Dept. of Agriculture and does not imply its approval to the exclusion of other products or vendors that may also be suitable. The cost of publishing this paper was defrayed in part by the payment of page charges. Under postal regulations, this paper therefore must be hereby marked advertisement solely to indicate this fact.
}

\section{Materials and Methods}

The rootstock seedlings (Table 1) were budded in a field nursery in Spring 1985 with the registered 'Hamlin' budline 815-XE F/D 5-4 obtained from the Florida Dept. of Agriculture and Consumer Services Budwood Registration Bureau,Winter Haven, Fla. The budded trees were planted on 10 Dec. 1986 at the test site near St. Cloud, Fla., with $4.2 \times 6.6-$ $\mathrm{m}$ spacing. There were six, three-tree replications on each rootstock arranged in randomized complete blocks. The site was previously in pasture; it was modified for planting by cutting and filling drainage ditches on two sides. The soil was mostly Myakka fine sand (sandy, siliceous, hyperthermic aeric haplaquods) at $\mathrm{pH}$ 5.5. The trees were irrigated with microsprinklers, and standard cultural practices were used, including chemical weed control within the rows and grass between the rows. Five to six fertilizer applications were hand-applied before the trees were 4 years old, and then three applications were made per year with a spreader, totaling $180 \mathrm{~kg}$ $\mathrm{N}$ and $149 \mathrm{~kg} \mathrm{~K} / \mathrm{ha}$ per year.

Yield records were taken at harvests on 5 Nov. 1990, 25 Nov. 1991, 12 Jan. 1992, and 2 Dec. 1993 when the trees were 4 to 7 years old. Yields per three-tree replication were measured by gauging the depth of fruit in standard 10-box tubs with a calibrated stick inserted in a crossbar placed over the tub. Each box equals $40.8 \mathrm{~kg}$ fruit.

Fruit quality was determined at each of the four harvests on 30-fruit samples by weighing them, extracting the juice with an electric reamer, and determining total soluble solids with a temperature-compensated refractometer and total acids by titration with $0.1560 \mathrm{~N}$
$\mathrm{NaOH}$. Juice color was measured with a chromameter (model CE 200; Minolta Camera Co., Osaka, Japan), which integrates tristimulus measurements into the color number used for grading juice. Peel color was determined by visually rating the fruit with a color chart (Harding et al., 1940). Two composite samples were collected at every harvest, one from the nine trees in replications 1 to 3 and one from the nine trees in replications 4 to 6 . Parallel 40 -fruit samples were processed in the commercial-type test facility of the Florida Dept. of Citrus, Lake Alfred. Tree height and width were measured at the last harvest, and the canopy volume was calculated by the formula (width ${ }^{2} \times$ height) $/ 4$. Width was limited by machine hedging, which kept the interrow space at $2.4 \mathrm{~m}$. The data were tested using an SAS program for analysis of variance; mean separation was by Duncan's multiple range test at $P \leq 0.05$.

\section{Results and Discussion}

The trees grew normally the first 3 years in the field and began to fruit in 1989, producing an estimated 3 to $35 \mathrm{~kg} /$ tree. The Christmas freeze in 1989 damaged the trees; most were defoliated, but trees on Vangasay lemon were damaged so severely that they were removed. Trees on HRS 920 (Smooth Flat Seville openpollinated hybrid) were stunted and unthrifty, and they also were eliminated. Trees on Nakorn pummelo x Flying Dragon trifoliate (HRS 898) also were variable in size, but they remained in the test. Five years after planting, all trees on Norton citrange (HRS 815) showed the $\mathrm{Zn}$ deficiency symptoms characteristic of citrus blight. Diagnostic tests, water injection with a syringe (water absorption in syringe injection tests: trees on Norton $32.7 \mathrm{ml} \cdot \mathrm{min}^{-1}$, healthy trees on Swingle $65.7 \mathrm{ml} \cdot \mathrm{min}^{-1}$ ) (Lee et al., 1984), and analysis of the outer trunk wood ( $\mathrm{Zn}$ in the outer trunk wood: Norton, 9 $\mu \mathrm{g} \cdot \mathrm{g}^{-1}$; Swingle, $4 \mu \mathrm{g} \cdot \mathrm{g}^{-1}$ ) (Wutscher et al., 1977) confirmed the diagnosis. The trees on Norton also were removed. Of the remaining trees on 13 rootstocks, four trees on Nakorn pummelo $x$ Flying Dragon trifoliate orange and two trees each on Changsha $x$ English small-flowered trifoliate orange (HRS 801) and Swingle (HRS 874) were lost to undetermined causes.

HRS 852 (Changsha mandarin $x$ English large-flowered trifoliate orange) emerged as the best rootstock in the test. A similar hybrid (FFI-131-20) performed well in an earlier test in southern Florida (Wutscher and Bistline, 1988). Trees on HRS 852 were of medium size (Table 1) and produced as much fruit as the much larger trees on HRS 802 (Siamese pummelo X Gotha Road trifoliate orange), but cumulative solids production per tree was equal for trees on HRS 802, HRS 852, and HRS 900 rootstocks (Table 2). Solids production determines the profitability of orange groves in Florida, where $90 \%$ of the oranges are processed. The growers get paid for the weight of solids delivered, not for the weight of fruit. Trees on Sacaton citrumelo (HRS 900) performed surprisingly well considering results 
from other locations (Gardner and Horanic, 1967; Wutscher, 1979), which were probably due to rigorous selection of presumed nucellar seedlings in the seedbed. Sacaton has $50 \%$ to $60 \%$ gametic seedlings, which when used as rootstocks, produces variable tree populations (Hutchison, 1977; Wutscher, 1979). Trees on the HRS 801 selection of the same parentage as HRS 852 and HRS 809 (Changsha $x$ English small-flowered trifoliate orange) were small and had the same high-yield efficiency (Table 1). These rootstocks would be appropriate for trees in high-density plantings. The trees on six rootstocks produced more fruit than those on Swingle citrumelo (Table 1).

The ranges of nine fruit quality factors were narrow (Table 3). Trees on Changsha $x$ English small-flowered trifoliate orange (HRS 801) and 81-17 citrumelo (HRS 931) produced some of the largest fruit, those on Sacaton citrumelo (HRS 900) and HRS 809 the smallest fruit. Fruit from trees on HRS 801 and HRS 811 had the lowest acid levels and corresponding high solids : acids ratios. Fruit from

Table 1. Tree height, tree volume, and yield efficiency of 'Hamlin' orange trees on 13 rootstocks.

\begin{tabular}{|c|c|c|c|c|c|c|}
\hline \multirow[b]{2}{*}{$\begin{array}{l}\mathrm{HRS}^{y} \\
\text { code }\end{array}$} & \multirow[b]{2}{*}{ Rootstock } & \multirow[b]{2}{*}{ Bionomials } & \multicolumn{2}{|c|}{ Tree size ${ }^{\mathrm{z}}$} & \multicolumn{2}{|c|}{1993 Yield efficiency ${ }^{2}$} \\
\hline & & & $\begin{array}{c}\mathrm{Ht} \\
(\mathrm{m})\end{array}$ & $\begin{array}{l}\text { Vol. } \\
\left(\mathrm{m}^{3}\right)\end{array}$ & $\begin{array}{c}\text { Fruit } \\
\left(\mathrm{kg} \cdot \mathrm{m}^{-3}\right)\end{array}$ & $\begin{array}{c}\text { Solids } \\
\left(\mathrm{kg} \cdot \mathrm{m}^{-3}\right)\end{array}$ \\
\hline$\overline{802}$ & $\begin{array}{l}\text { Siamese pummelo } \times \text { Gotha } \\
\text { Road trifoliate orange }\end{array}$ & $\begin{array}{l}\text { Citrus grandis (L.) Osb. } \mathrm{X} \\
\quad \text { Poncirus trifoliata L. Raf. }\end{array}$ & $4.0 \mathrm{a}$ & $17.7 \mathrm{a}$ & $13.9 \mathrm{~d}$ & $0.7 \mathrm{e}$ \\
\hline 852 & $\begin{array}{l}\text { Changsha mandarin } \times \text { English } \\
\text { large-flowered trifoliate orange }\end{array}$ & $\begin{array}{l}\text { C. reticulata Blanco X } \\
\text { P. trifoliata }\end{array}$ & $3.0 \mathrm{~cd}$ & $11.4 \mathrm{~cd}$ & $20.7 \mathrm{~b}$ & $1.1 \mathrm{~b}$ \\
\hline 900 & Sacaton citrumelo & P. trifoliata X. C. paradisi Macf. & $3.6 \mathrm{~b}$ & $14.5 \mathrm{~b}$ & $15.9 \mathrm{~cd}$ & $0.8 \mathrm{de}$ \\
\hline 902 & $\begin{array}{l}\text { Mott grapefruit } \times \text { Gotha Road } \\
\text { trifoliate orange (citrumelo) }\end{array}$ & C. paradisi $\times$ P. trifoliata & $3.2 \mathrm{c}$ & $11.9 \mathrm{c}$ & $19.7 \mathrm{~b}$ & $1.0 \mathrm{bc}$ \\
\hline 886 & W-4 citrumelo & C. paradisi $\times$ P. trifoliata & $3.7 \mathrm{~b}$ & $15.1 \mathrm{~b}$ & $15.0 \mathrm{~d}$ & $0.8 \mathrm{de}$ \\
\hline 801 & $\begin{array}{l}\text { Changsha mandarin } \mathrm{x} \\
\text { English small-flowered trifoliate orange }\end{array}$ & C. reticulata $\times$. $P$. trifoliata & $2.5 \mathrm{fg}$ & $6.8 \mathrm{gh}$ & $25.3 \mathrm{a}$ & $1.3 \mathrm{a}$ \\
\hline 874 & Swingle citrumelo & C. paradisi $\times P$. trifoliata & $3.0 \mathrm{~cd}$ & $9.1 \mathrm{ef}$ & $20.2 \mathrm{~b}$ & $1.0 \mathrm{bc}$ \\
\hline 809 & $\begin{array}{l}\text { Changsha mandarin } x \\
\text { English large-flowered trifoliate orange }\end{array}$ & C. reticulata Blanco $\times$ P. trifoliata & $2.6 \mathrm{fg}$ & $7.1 \mathrm{f}-\mathrm{h}$ & $24.0 \mathrm{a}$ & $1.3 \mathrm{a}$ \\
\hline 896 & Cleopatra mandarin $\mathrm{x}$ & & & & & \\
\hline & Rubidou trifoliate orange & C. reticulata $\times P$. trifoliata & $2.6 \mathrm{fg}$ & $8.4 \mathrm{f}-\mathrm{h}$ & $19.0 \mathrm{~b}$ & $1.1 \mathrm{~b}$ \\
\hline 898 & $\begin{array}{l}\text { Nakorn pummelo } \mathrm{x} \\
\text { Flying Dragon trifoliate orange }\end{array}$ & C. grandis $\times P$. trifoliata & 2.7 ef & $7.5 \mathrm{f}-\mathrm{h}$ & $20.1 \mathrm{~b}$ & $1.1 \mathrm{~b}$ \\
\hline 811 & $\begin{array}{l}\text { Smooth Flat Seville } x \\
\text { Swingle citrumelo }\end{array}$ & $\begin{array}{l}\text { C. aurantium } \mathrm{L} \text {. hybrid? } \mathrm{x} \\
(\text { C. paradisi } \times \text { P. trifoliata })\end{array}$ & 2.7 ef & $8.9 \mathrm{e}-\mathrm{g}$ & $18.4 \mathrm{bc}$ & $0.9 \mathrm{c}-\mathrm{e}$ \\
\hline 931 & $\begin{array}{l}\text { 81-17 citrumelo, Duncan grapefruit } x \\
\text { trifoliate orange }\end{array}$ & C. paradisi $\times P$. trifoliata & $2.9 \mathrm{de}$ & $9.9 \mathrm{de}$ & $14.2 \mathrm{~d}$ & $0.8 \mathrm{~d}$ \\
\hline 897 & $\begin{array}{l}\text { Cleopatra mandarin } \mathrm{x} \\
\text { Flying Dragon trifoliate orange }\end{array}$ & C. reticulata $\times P$. trifoliata & $2.4 \mathrm{~g}$ & $6.6 \mathrm{~h}$ & $20.2 \mathrm{~b}$ & $1.1 \mathrm{~b}$ \\
\hline
\end{tabular}

${ }^{2}$ Mean separation within columns by Duncan's multiple range test at $P \leq 0.05$.

${ }^{y}$ A code system used at the U.S. Horticultural Research Laboratory, Orlando, Fla.

Table 2. Fruit and solids yield of 'Hamlin' orange trees on 13 rootstocks.

\begin{tabular}{|c|c|c|c|c|c|c|c|c|c|c|c|}
\hline \multirow[b]{2}{*}{$\begin{array}{l}\text { HRS } \\
\text { code }^{y}\end{array}$} & \multirow[b]{2}{*}{ Rootstocks } & \multicolumn{5}{|c|}{$\mathrm{kg}$ fruit/tree ${ }^{z}$} & \multicolumn{5}{|c|}{ kg solids/tree ${ }^{z}$} \\
\hline & & 1990-91 & $1991-92$ & $1992-93$ & 1993-94 & $\begin{array}{c}\text { Cumulative } \\
\text { yield }\end{array}$ & 1990-91 & $1991-92$ & 1992-93 & 1993-94 & $\begin{array}{c}\text { Cumlative } \\
\text { yield }\end{array}$ \\
\hline 802 & $\begin{array}{l}\text { Siamese pummelo } \times \text { Gotha } \\
\text { Road trifoliate orange }\end{array}$ & $98.7 \mathrm{bc}$ & $142.2 \mathrm{ab}$ & $295.2 \mathrm{a}$ & $245.0 \mathrm{a}$ & $781.1 \mathrm{a}$ & $1.4 \mathrm{~b}-\mathrm{d}$ & $6.6 \mathrm{~b}$ & $14.8 \mathrm{a}$ & $11.8 \mathrm{ab}$ & $34.6 \mathrm{ab}$ \\
\hline 852 & $\begin{array}{l}\text { Changsha mandarin } \mathrm{x} \\
\text { English large-flowered } \\
\text { trifoliate orange }\end{array}$ & $108.2 \mathrm{~b}$ & $153.8 \mathrm{a}$ & $272.0 \mathrm{a}$ & $235.3 \mathrm{ab}$ & $769.2 \mathrm{a}$ & $1.7 \mathrm{ab}$ & $7.7 \mathrm{a}$ & $14.8 \mathrm{a}$ & $12.4 \mathrm{a}$ & $36.6 \mathrm{a}$ \\
\hline 900 & Sacaton citrumelo & $98.7 \mathrm{bc}$ & $133.3 \mathrm{a}-\mathrm{c}$ & $274.2 \mathrm{a}$ & $225.3 \mathrm{ab}$ & $731.5 \mathrm{ab}$ & $1.6 \mathrm{bc}$ & $6.3 \mathrm{bc}$ & $14.0 \mathrm{a}$ & $11.8 \mathrm{ab}$ & $33.7 \mathrm{ab}$ \\
\hline 902 & $\begin{array}{l}\text { Mott grapefruit } x \text { Gotha } \\
\text { Road trifoliate orange } \\
\text { citrumelo }\end{array}$ & $80.8 \mathrm{~b}-\mathrm{d}$ & $132.7 \mathrm{a}-\mathrm{d}$ & $223.8 \mathrm{~b}$ & $221.2 \mathrm{~b}$ & $658.5 \mathrm{c}$ & $1.0 \mathrm{de}$ & $6.1 \mathrm{~b}-\mathrm{d}$ & $11.4 \mathrm{bc}$ & $11.1 \mathrm{~b}$ & $29.6 \mathrm{~cd}$ \\
\hline 886 & W-4 citrumelo & $70.7 \mathrm{c}-\mathrm{e}$ & $110.7 \mathrm{c}-\mathrm{e}$ & $232.7 \mathrm{~b}$ & $226.7 \mathrm{ab}$ & $640.8 \mathrm{c}$ & $1.0 \mathrm{de}$ & $5.3 \mathrm{c}-\mathrm{e}$ & $13.3 \mathrm{ab}$ & $11.8 \mathrm{ab}$ & $31.4 \mathrm{bc}$ \\
\hline 801 & $\begin{array}{l}\text { Changsha mandarin } \mathrm{x} \\
\text { English small-flowered } \\
\text { trifoliate orange }\end{array}$ & $165.8 \mathrm{a}$ & $110.0 \mathrm{c}-\mathrm{e}$ & $173.5 \mathrm{~cd}$ & $172.2 \mathrm{~cd}$ & $621.5 \mathrm{c}$ & $2.1 \mathrm{a}$ & $4.8 \mathrm{de}$ & $9.0 \mathrm{de}$ & $8.6 \mathrm{c}-\mathrm{e}$ & $24.5 \mathrm{e}-\mathrm{g}$ \\
\hline 874 & Swingle citrumelo & $89.7 b-d$ & $122.0 \mathrm{~b}-\mathrm{d}$ & $204.3 \mathrm{bc}$ & $178.2 \mathrm{c}$ & $594.2 \mathrm{~d}$ & $1.5 \mathrm{bc}$ & $6.0 \mathrm{~b}-\mathrm{e}$ & $11.5 \mathrm{bc}$ & $9.2 \mathrm{c}$ & $28.2 \mathrm{c}-\mathrm{e}$ \\
\hline 809 & $\begin{array}{l}\text { Changsha mandarin } x \\
\text { English large-flowered } \\
\text { trifoliate orange }\end{array}$ & $75.5 \mathrm{c}-\mathrm{e}$ & $111.5 \mathrm{c}-\mathrm{e}$ & $177.5 \mathrm{~cd}$ & $171.7 \mathrm{de}$ & $536.2 \mathrm{de}$ & $1.3 \mathrm{~b}-\mathrm{d}$ & $5.7 \mathrm{~b}-\mathrm{e}$ & $9.9 \mathrm{c}-\mathrm{e}$ & $9.2 \mathrm{c}$ & $26.3 \mathrm{~d}-\mathrm{f}$ \\
\hline 896 & $\begin{array}{l}\text { Cleopatra mandarin } \mathrm{x} \\
\text { Rubidou trifoliate orange }\end{array}$ & $85.7 \mathrm{~b}-\mathrm{d}$ & $114.8 \mathrm{c}-\mathrm{e}$ & $175.5 \mathrm{~cd}$ & $156.7 \mathrm{c}-\mathrm{e}$ & $532.7 \mathrm{de}$ & $1.4 \mathrm{~b}-\mathrm{d}$ & $5.7 \mathrm{~b}-\mathrm{e}$ & $10.3 \mathrm{~cd}$ & $9.0 \mathrm{~cd}$ & $26.4 \mathrm{~d}-\mathrm{f}$ \\
\hline 898 & $\begin{array}{l}\text { Nakorn pummelo } \mathrm{x} \\
\text { Flying Dragon } \\
\text { trifoliate orange }\end{array}$ & $95.2 \mathrm{~b}-\mathrm{d}$ & 97.3 ef & $172.8 \mathrm{~cd}$ & $149.0 \mathrm{~d}-\mathrm{f}$ & $514.3 \mathrm{de}$ & $1.5 \mathrm{bc}$ & $5.4 \mathrm{c}-\mathrm{e}$ & $9.6 \mathrm{c}-\mathrm{e}$ & $8.1 \mathrm{c}-\mathrm{e}$ & $24.6 \mathrm{e}-\mathrm{g}$ \\
\hline 811 & $\begin{array}{c}\text { Smooth Flat Seville } x \\
\text { Swingle citrumelo }\end{array}$ & $49.0 \mathrm{e}$ & $75.3 \mathrm{f}$ & $177.0 \mathrm{~cd}$ & $161.8 \mathrm{dc}$ & $463.1 \mathrm{e}$ & $0.6 \mathrm{e}$ & $3.3 \mathrm{e}$ & $8.5 \mathrm{de}$ & $7.8 \mathrm{ef}$ & $20.2 \mathrm{~h}$ \\
\hline 931 & $\begin{array}{l}\text { 81-17 citrumelo, Duncan } \\
\text { grapefruit } x \text { trifoliate orange }\end{array}$ & $64.5 \mathrm{de}$ & 95.8 ef & $163.8 \mathrm{~d}$ & 138.7 ef & $462.8 \mathrm{e}$ & $1.1 \mathrm{~cd}$ & $4.8 \mathrm{e}$ & $8.2 \mathrm{de}$ & 7.9 ef & $23.0 \mathrm{f}-\mathrm{h}$ \\
\hline 897 & $\begin{array}{l}\text { Cleopatra mandarin } \mathrm{x} \\
\text { Flying Dragon trifoliate } \\
\text { orange }\end{array}$ & $66.0 \mathrm{de}$ & $108.8 \mathrm{de}$ & $153.8 \mathrm{~d}$ & $133.3 \mathrm{f}$ & $461.9 \mathrm{e}$ & $1.0 \mathrm{de}$ & $5.3 \mathrm{c}-\mathrm{e}$ & $8.0 \mathrm{e}$ & $7.0 \mathrm{f}$ & $21.3 \mathrm{gh}$ \\
\hline
\end{tabular}

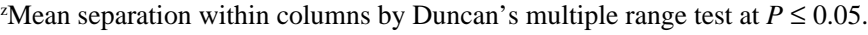

${ }^{y}$ A code system used at the U.S. Horticultural Research Laboratory, Orlando, Fla. 


\begin{tabular}{|c|c|c|c|c|c|c|c|c|c|c|}
\hline $\begin{array}{l}\text { HRS } \\
\text { code }^{y}\end{array}$ & Rootstocks & $\begin{array}{l}\text { Fruit } \\
\text { wt } \\
(\mathrm{g})\end{array}$ & $\begin{array}{l}\text { Fruit } \\
\text { diam } \\
(\mathrm{cm})\end{array}$ & $\begin{array}{l}\text { Peel } \\
\text { color }^{\mathrm{x}}\end{array}$ & $\begin{array}{l}\text { Rind } \\
\text { thickness } \\
(\mathrm{mm})\end{array}$ & $\begin{array}{l}\text { Juice } \\
\text { content } \\
(\%)\end{array}$ & $\begin{array}{c}\text { Total } \\
\text { soluble } \\
\text { solids } \\
(\%)\end{array}$ & $\begin{array}{l}\text { Total } \\
\text { acids } \\
(\%)\end{array}$ & TSS : TA $^{w}$ & $\begin{array}{l}\text { Juice } \\
\text { color }^{v}\end{array}$ \\
\hline 802 & $\begin{array}{l}\text { Siamese pummelo } \times \text { Gotha } \\
\text { Road trifoliate orange }\end{array}$ & $169 \mathrm{~b}-\mathrm{d}$ & $7.0 \mathrm{~b}^{\mathrm{u}}$ & I & $3.5 \mathrm{ab}$ & $49.0 \mathrm{~b}-\mathrm{d}$ & $9.0 \mathrm{fg}$ & $0.73 \mathrm{e}$ & $12.3 \mathrm{c}-\mathrm{e}$ & $33.5 \mathrm{a}$ \\
\hline 852 & $\begin{array}{l}\text { Changsha mandarin } \mathrm{x} \\
\text { English large-flowered } \\
\text { trifoliate orange }\end{array}$ & $172.0 \mathrm{bc}$ & $7.0 \mathrm{~b}$ & $\mathrm{H}$ & $3.4 \mathrm{a}-\mathrm{c}$ & $50.2 \mathrm{a}$ & $9.6 \mathrm{~cd}$ & $0.79 \mathrm{~d}$ & $12.2 \mathrm{c}-\mathrm{e}$ & $33.3 \mathrm{a}-\mathrm{c}$ \\
\hline 900 & Sacaton citrumelo & $162.5 \mathrm{~cd}$ & $6.8 \mathrm{c}$ & $\mathrm{H}$ & $3.4 \mathrm{a}-\mathrm{c}$ & $48.7 \mathrm{~cd}$ & $9.5 \mathrm{de}$ & $0.79 \mathrm{~d}$ & $12.0 \mathrm{e}$ & $33.4 \mathrm{ab}$ \\
\hline 902 & $\begin{array}{l}\text { Mott grapefruit } \times \text { Gotha Road } \\
\text { trifoliate orange citrumelo }\end{array}$ & $181.5 \mathrm{ab}$ & $7.1 \mathrm{ab}$ & $\mathrm{H}$ & $3.7 \mathrm{a}$ & $48.3 \mathrm{~d}$ & $9.2 \mathrm{ef}$ & $0.75 \mathrm{e}$ & $12.3 \mathrm{c}-\mathrm{e}$ & $33.3 \mathrm{a}-\mathrm{c}$ \\
\hline 886 & W-4 citrumelo & $177.3 \mathrm{~b}$ & $7.0 \mathrm{~b}$ & $\mathrm{H}$ & $3.5 \mathrm{ab}$ & $48.6 \mathrm{~cd}$ & $9.6 \mathrm{~cd}$ & $0.84 \mathrm{a}$ & $11.4 \mathrm{f}$ & $33.4 \mathrm{ab}$ \\
\hline 801 & $\begin{array}{l}\text { Changsha mandarin } x \\
\text { English small-flowered } \\
\text { trifoliate orange }\end{array}$ & $190.6 \mathrm{a}$ & $7.3 \mathrm{a}$ & $\mathrm{H}$ & $3.7 \mathrm{a}$ & $46.5 \mathrm{e}$ & $9.2 \mathrm{ef}$ & $0.70 \mathrm{f}$ & $13.1 \mathrm{a}$ & $33.3 \mathrm{a}-\mathrm{c}$ \\
\hline 874 & Swingle citrumelo & $173.8 \mathrm{bc}$ & $7.0 \mathrm{~b}$ & $\mathrm{H}$ & $3.4 \mathrm{a}-\mathrm{c}$ & $49.2 \mathrm{~b}-\mathrm{d}$ & $9.7 \mathrm{~b}-\mathrm{d}$ & $0.80 \mathrm{~cd}$ & $12.1 \mathrm{de}$ & $33.2 \mathrm{a}-\mathrm{c}$ \\
\hline 809 & $\begin{array}{l}\text { Changsha mandarin } x \\
\text { English large-flowered } \\
\text { trifoliate orange }\end{array}$ & $159.6 \mathrm{~d}$ & $6.8 \mathrm{c}$ & $\mathrm{H}$ & $3.2 \mathrm{c}$ & $49.0 \mathrm{~b}-\mathrm{d}$ & $10.0 \mathrm{ab}$ & $0.82 \mathrm{a}-\mathrm{c}$ & $12.0 \mathrm{e}$ & $33.2 \mathrm{a}-\mathrm{c}$ \\
\hline 896 & $\begin{array}{l}\text { Cleopatra mandarin } x \\
\text { Rubidou trifoliate orange }\end{array}$ & $174.5 \mathrm{~b}$ & $7.0 \mathrm{~b}$ & $\mathrm{H}$ & $3.3 \mathrm{bc}$ & $49.9 \mathrm{ab}$ & $10.1 \mathrm{a}$ & $0.80 \mathrm{~cd}$ & $12.6 \mathrm{~b}-\mathrm{d}$ & $33.1 \mathrm{bc}$ \\
\hline 898 & $\begin{array}{l}\text { Nakorn pummelo } \times \text { Flying } \\
\text { Dragon trifoliate orange }\end{array}$ & $177.5 \mathrm{~b}$ & $7.1 \mathrm{ab}$ & I & $3.5 \mathrm{ab}$ & $49.3 \mathrm{a}-\mathrm{c}$ & $9.9 \mathrm{ab}$ & $0.81 \mathrm{~b}-\mathrm{d}$ & $12.2 \mathrm{c}-\mathrm{e}$ & $33.2 \mathrm{a}-\mathrm{c}$ \\
\hline 811 & $\begin{array}{l}\text { Smooth Flat Seville } x \\
\text { Swingle citrumelo }\end{array}$ & $176.9 \mathrm{~b}$ & $7.1 \mathrm{ab}$ & I & $3.5 \mathrm{ab}$ & $46.9 \mathrm{e}$ & $8.9 \mathrm{~g}$ & $0.68 \mathrm{f}$ & $13.1 \mathrm{a}$ & $33.3 \mathrm{a}-\mathrm{c}$ \\
\hline 931 & $\begin{array}{l}\text { 81-17 citrumelo, Duncan } \\
\text { grapefruit } x \text { trifoliate orange }\end{array}$ & $191.8 \mathrm{a}$ & $7.3 \mathrm{a}$ & $\mathrm{H}$ & $3.7 \mathrm{a}$ & $49.8 \mathrm{ab}$ & $9.9 \mathrm{ab}$ & $0.83 a b$ & $11.9 \mathrm{e}$ & $33.0 \mathrm{c}$ \\
\hline 897 & $\begin{array}{l}\text { Cleopatra mandarin } x \text { Flying } \\
\text { Dragon trifoliate orange }\end{array}$ & $175.4 \mathrm{~b}$ & $7.1 \mathrm{ab}$ & $\mathrm{H}$ & $3.3 \mathrm{bc}$ & $49.5 \mathrm{a}-\mathrm{c}$ & $9.4 \mathrm{de}$ & $0.74 \mathrm{e}$ & $12.7 \mathrm{bc}$ & $33.3 \mathrm{a}-\mathrm{c}$ \\
\hline
\end{tabular}

${ }^{2}$ Mean of two composite 30-fruit samples (replications 1 to 3 and 4 to 6 ) collected at each of four harvests in 1990-93.

${ }^{y}$ A code system used at the U.S. Horticultural Research Laboratory, Orlando, Fla.

xPeel color according to color tables in Harding et al. (1940); colors range from A (completely green) to L (dark red).

wTSS : TA = total soluble solids : total acid ratio.

vJuice color as color number determined with chromameter (model CE 200; Minolta Camera Co., Osaka, Japan).

"Mean separation within columns by Duncan's multiple range test at $P \leq 0.05$.

trees on HRS 852, HRS 896, HRS 898, HRS 931, and HRS 897 had the highest juice content. Comparing the juice content obtained by hand-reaming and by commercial extracting machines in the Dept. of Citrus Laboratory (data not shown) indicated that $5 \%$ to $10 \%$ more juice was extracted in the test house. This procedure also increased kilogram solids per cubic meter of canopy volume and the weight of solids produced per tree to levels greater than those reported in Tables 1 and 2, more closely resembling those that would be attained in commercial production. There was little difference in juice color due to rootstock (Table 3); juice color from fruit on trees on all rootstocks was typical for 'Hamlin' orange and well below color number 36 , required for grade A juice.

All of the rootstocks in the experiment were tested previously as plants in pots (Grimm and Hutchison, 1973; Hutchison, 1985) and found to be tolerant to Phytophthora parasitica Dastur and tristeza. Also, the experimental site was tristeza-infected. The trees on Norton citrange developed citrus blight early; little can be said of the potential tolerance to this decline of the 12 rootstocks other than Swingle (Castle et al., 1988), which is resistant. HRS
852 (Changsha mandarin x English largeflowered trifoliate orange) likely will be released for commercial use as soon as seed sources can be established. Most of Florida's soils are sandy and acidic, as in our test plot; therefore, similar results can be expected in much of the state's citrus areas.

\section{Literature Cited}

Castle, W.S., D.P.H. Tucker, A.H. Krezdorn, and C.O. Youtsey. 1993. Rootstocks for Florida citrus. 2nd ed. Special Publ. 42, Univ. of Florida Inst. of Food and Agricultural Sciences, Gainesville.

Castle, W.S., H.K. Wutscher, C.O. Youtsey, and R.R. Pelosi. 1988. Citrumelos as rootstocks for Florida citrus. Proc. Fla. State Hort. Soc. 101:2833.

Gardner, F.E. and G.E. Horanic. 1967. Poncirus trifoliata and some of its hybrids as rootstocks for Valencia sweet orange. Proc. Fla. State Hort. Soc. 80:85-88.

Grimm, G.R. and D.J. Hutchison. 1973. A procedure for evaluating resistance of citrus seedlings to Phytophthora parasitica. Plant Dis. Rptr. 57:669-672.

Harding, P.L., J.R. Winston, and D.F. Fisher. 1940. Seasonal changes in Florida oranges. Tech. Bul. 753, U.S. Dept. of Agr., Washington, D.C.
Hutchison, D.J. 1977. Influence of rootstock on the performance of 'Valencia' sweet orange. Proc. Intl. Soc. Citricult. 2:523-525.

Hutchison, D.J. 1985. Rootstock development screening and selection for disease tolerance and horticultural characteristics. Fruit Var. J. 29:21-25.

Lee, R.F., L.J. Marais, L.W. Timmer, and J.H. Graham. 1984. Syringe injection of water into the trunk: A rapid diagnostic test for citrus blight. Plant Dis. 68:511-513.

Rouse, R.E. and H.K. Wutscher. 1985. Heavy soil and bud union crease with some grapefruit clones limit use of Swingle citrumelo rootstock. HortScience 20:259-261.

Wutscher, H.K. 1979. Citrus rootstocks, p. 237269. In: J. Janick (ed.). Horticultural reviews vol. 1. AVI, Westport, Conn.

Wutscher, H.K. 1988. The rootstock situation in Florida, p. 67-73. In: R. Goren and K. Mendel (eds.). Proc. Sixth Intl. Citrus Congr. vol. 1. Tel Aviv, Israel.

Wutscher, H.K. and F.W. Bistline. 1988. Performance of 'Hamlin' orange on 30 citrus rootstocks in southern Florida. J. Amer. Soc. Hort. Sci. 113:493-497.

Wutscher, H.K., M. Cohen, and R.H. Young. 1977. Zinc and water soluble phenolic levels in the wood for diagnosis of citrus blight. Plant Dis. Rptr. 61:572-574. 